\title{
Effects of the Combination of High-intensity Interval Training and Ecdysterone on Learning and Memory Abilities, Antioxidant Enzymes Activities, and Neuronal Population in an Amyloid-beta-induced Rat Model of Alzheimer's Disease
}

\section{Parsa Gholipour}

Hamadan University of Medical Sciences Medical School

alireza komaki ( $\triangle$ parsa1996gh@gmail.com )

Hamadan University of Medical Sciences https://orcid.org/0000-0002-2172-3043

Mahdi Ramezani

Hamadan University of Medical Sciences

\section{Research Article}

Keywords: Amyloid-beta (AB), Alzheimer's disease (AD), Ecdysterone (Ecdy), High-intensity interval training (HIIT), Cognitive decline, Oxidative stress

Posted Date: January 12th, 2022

DOI: https://doi.org/10.21203/rs.3.rs-983963/v2

License: (9) This work is licensed under a Creative Commons Attribution 4.0 International License. Read Full License

Version of Record: A version of this preprint was published at Physiology \&amp; Behavior on April 1st, 2022. See the published version at https://doi.org/10.1016/j.physbeh.2022.113817. 


\section{Abstract}

Aims: Oxidative stress and neuronal death are the primary reasons for the progression of amyloid-beta $(A B)$ deposition and cognitive deficits in Alzheimer's disease (AD). Ecdysterone (Ecdy), a common derivative of ecdysteroids, possesses free radical scavenging and cognitive-improving effects. Highintensity interval training (HIIT) may be a therapeutic strategy for improving cognitive decline and oxidative stress. The present study was aimed to evaluate the effect of HIIT alone and its combination with Ecdysterone on the changes in learning and memory functions, hippocampal antioxidant enzymes activities, and neuronal population after $A D$ induced by $A \beta$ in male rats.

Materials and methods: Following ten days of Aß-injection, HIIT exercise and Ecdysterone treatment (10 $\mathrm{mg} / \mathrm{kg} /$ day; P.O.) were initiated and continued for eight consecutive weeks in rats. At the end of the treatment period, rat's learning and memory functions were assessed using water-maze and passiveavoidance tests. Moreover, the activity of superoxide dismutase (SOD), catalase (CAT), Glutathione Peroxidase (GPx), Glutathione Reductase (GRx) and neuronal population were evaluated in rat's brains.

Results: The results indicated that $A \beta$ injection disrupted spatial/passive avoidance learning and memory in both water-maze and passive-avoidance paradigms, accompanied by a decrease in the superoxide dismutase and catalase (as endogenous antioxidants) in rat hippocampus. Additionally, $A \beta$ injection resulted in neuronal loss in the cerebral cortex and hippocampus. Although consumption of Ecdysterone separately improved spatial/passive avoidance learning and memory impairments, recovered hippocampal activity of SOD, CAT, GRx, GRx and prevented the hippocampal neuronal loss, its combination with HIIT resulted in a more powerful and effective amelioration in all the above-mentioned A $\beta$-neuropathological changes.

Conclusion: The current work's data confirms that a combination of HIIT exercise and Ecdysterone treatment could be a promising potential therapeutic agent against AD-associated cognitive decline, owing to their free radical scavenging and neuroprotective properties.

\section{Introduction}

It is proven that Alzheimer's disease (AD) is the most potent neurodegenerative ailment (1) that includes about $6 \%$ or $7 \%$ of the elderly population over 65 years old (more than forty million people worldwide) that chance of prevalence after this age becomes doubles every five years (2). AD is characterized clinically by progressive cognitive dysfunctions and behavioral disorders related to the lack or decrease of synaptic connections and, of course, neuronal apoptosis as a result of progressive aggregation of amyloid- $\beta$ (AB) and hyperphosphorylation of Tau protein in the hippocampus and cerebral cortex that makes patients confused and occasionally they have difficulty in their routine life. Also, oxidative stress is a critical component of pathogenesis in $\operatorname{AD}(3,4)$, so that there are studies that heretofore have reported the rise of oxidative stress markers in the brain tissue of AD patients, parameters such as lipid peroxidation, protein oxidation, and formation of reactive oxygen species (ROS) $(5,6)$. meanwhile, the 
hippocampus area has a crucial role in learning and memory (7). It is one of the most vulnerable regions in the brain, which is prone to succumb to oxidative stress derided by dementia (8). Nowadays, it is unclear whether oxidative stress is a significant reason or rises from mitochondrial dysfunctions associated with $A D(9)$.

It is reported that supplementations with antioxidant properties are helpful, particularly in the early stages of disease (10). At the same time, an array of natural antioxidants have been drawn from plants, including phytoestrogens, which can alleviate oxidative stress (11,12). Ecdysterone (Ecdy) (Fig.1) is one of the components of phytoestrogen that is an insect hormone and provides regulating with the molting, metamorphosis and reproduction of arthropods (13). Besides, it is widespread among a considerable number of plants with a high concentration in some certain plants such as; (Achyranthes bidentate) and (Cyanotis arachnoidea) $(14,15)$. In addition, evidence investigates that Ecdy may have substantially positive pharmacological impacts in mammals, such as; stimulating protein synthesis $(16,17)$, promoting carbohydrate and lipid metabolism (18), preserving apoptosis (19), affecting the CNS as well as enhancing immunomodulation. In other words, Ecdy is found by increasing the number of medical worth, also, the anti-oxidation profits of Ecdy have been reported (20). Besides, previous studies suggested that Ecdy can promote learning, memory and orally treat cognitive dysfunctions of rats induced by beta-amyloid peptide fragment ${ }_{25-35}\left(A \beta_{25-35}\right)$ and raise the expression of $C$-fos. This gene is an indicator for neuronal activity and firmly has relation with learning and memory in cortex and hippocampus (21). Likewise, earlier studies in terms of behavioral investigations have indicated that Ecdy caused shorter latency and searching distance in Morris water maze test between A $\beta$-induced rats that means Ecdy could improve impaired spatial memory (22).

On the other hand, surveys have shown that the administration of exercise training (ET) can cause an improvement of passive avoidance learning and spatial memory in rodents $(23,24)$. Furthermore, ET accomplishment increases hippocampal Neurogenesis, synaptic plasticity, as well, neurotransmission in the hippocampus that on their own can contribute to cognitive advancement $(25,26)$. However, never should it be forgotten that there is no supporting evidence to illustrate which combination of time, frequency, intensity, and generally type of exercise may have a more significant effect on cognitive niches. Based on studies, High-Intensity Interval Training (HIIT) through increases in $\mathrm{H}_{2} \mathrm{O}_{2}$ and TNF-a can lead to a significant improvement in BDNF (Brain-Derived Neurotrophic Factor) and GDNF (Glial Derived Neurotrophic Factor) concentrations (scientists notice these factors as indexes for memory and learning). Thus, training with interval bouts that need maximum effort will result in better neurotrophic gain (27). Additionally, this training method regulates hippocampal oxidative stress, BDNF, and inflammatory mediators in laboratory animals. At the behavioral level, it has been shown that HIIT can reduce learning decline in the Morris water maze test in rats with $\operatorname{AD}(28,29)$.

Although many remedial methods, including consuming chemical and herbal medicine and different ways of physical activity to inhibit AD progress, are available, an effective treatment against $A D$ still does not exist $(30,31)$. So, in the present study, we decided to determine the synchronous effect of HIIT 
accompanied by Ecdy in memory, learning, and cognitive functions in male Wistar rats induced by betaamyloid.

\section{Methods}

\subsection{Animal study}

Before starting the experiment, seventy-two adult male Wistar rats (weighing 175-200 gr) were purchased from the breeding institute of the animal house of Hamadan University of Medical Sciences (UMSHA). The animals were housed tree in a cage in a temperature-controlled room with $23-25^{\circ} \mathrm{C}$ and $50-70 \%$ relative humidity under a 12:12 light-dark cycle from 19 till seven that treatment protocol and behavioral tests started in light cycles. Also, every animal accessed to food (rodent pellets consisted of $23 \%$ protein, $47 \%$ carbohydrate, $5 \%$ lipids, $5 \%$ cellulose, $20 \%$ water, and vitamins and minerals with a caloric density of approximately $3.0 \mathrm{kcal} / \mathrm{g}$ ) (32) and water freely. All experimental processes and animal care procedures were confirmed by the Veterinary Ethics Committee of UMSHA and were confirmed with the Guidelines of the principles of laboratory animal care in the National Center of Health (code: IR.UMSHA.REC.1400.458).

\subsection{Experimental design}

At first, Rats for seven days were domesticated using handling and accustoming to circumstances. Then they were randomly assigned into nine groups(n=8 per group): (group1) control health (Control); they received saline 9\% (as Ecdy dissolvent) for eight weeks through oral gavage (33). (group2) Sham; they received a five $\mu \mathrm{L}$ vehicle of beta-amyloid (phosphate-buffered saline (PBS)) via an intrahippocampal injection after stereotaxic surgery. (group3) Ecdy; they received Ecdy $(10 \mathrm{mg} / \mathrm{kg} / \mathrm{day})$ for eight weeks through oral gavage(33). (group4) HIIT; they performed HIIT on a special treadmill for eight weeks (5 sessions per week) (34). (group5) Ecdy + HIIT; they received Ecdy (10mg/kg/day) for eight weeks through oral gavage also performed HIIT on a treadmill for eight weeks. (group6) control Alzheimer's (Alzheimer's); they received five $\mu \mathrm{L}$ beta-amyloid via an intrahippocampal injection after stereotaxic surgery, then received saline $9 \%$ for eight weeks through oral gavage (35). (group7) Alzheimer + Ecdy; they received Ecdy $(10 \mathrm{mg} / \mathrm{kg} /$ day) for eight weeks through oral gavage after receiving five $\mu \mathrm{L}$ betaamyloid via intrahippocampal injection. (group8) Alzheimer + HIIT; they performed HIIT on a special treadmill for eight weeks after receiving five $\mu \mathrm{L}$ beta-amyloid via intrahippocampal injection. (9) Alzheimer + Ecdy + HIIT; they received Ecdy $(10 \mathrm{mg} / \mathrm{kg} /$ day $)$ for eight weeks through oral gavage also performed HIIT on a treadmill for eight weeks after receiving five $\mu \mathrm{L}$ beta-amyloid via intrahippocampal injection (Fig.2). Although studies also have reported some other dosages such as 1 and $100 \mathrm{mg} / \mathrm{kg} / \mathrm{day}$, with due attention to studies, we decided to use $10 \mathrm{mg} / \mathrm{kg} /$ day of Ecdy as an efficient dosage (33).

\subsection{A $\beta$ preparation, injection and stereotaxic surgery}

The Amyloid- $\beta_{1-42}$ peptide $(100 \mu \mathrm{L}$ ) (cat number SCP0038, bought from Sigma Aldrich, USA) dissolved in $100 \mu \mathrm{L}$ PBS (as vehicle solution) then incubated at $37^{\circ} \mathrm{C}$ for seven days before in vivo utilizing. This action leads to fibril formation, which has neurotoxic properties (36). After preparing $A \beta_{1-42}$ to induce $A D$ 
model, the rats were anesthetized with intraperitoneal (i.p) injection of ketamine $(100 \mathrm{mg} / \mathrm{kg})$ and xylzine $(10 \mathrm{mg} / \mathrm{kg})$. Afterward, rats were stuck in the stereotaxic apparatus (Stoelting Co., Wood Dale, IL, USA), and their scalp was split. Then Bregma and Lambda adjusted to balance level in terms of the horizontal plane and a hole drilled above the ventricular area in the skull surface (coordinates: AP: $-1.2 \mathrm{~mm}$ posterior to the Bregma, ML, $\pm 2 \mathrm{~mm}$ lateral, and DV: $4.0 \mathrm{~mm}$ below the dura)(37). The injection was conducted with a five $\mu \mathrm{L}$ microsyringe (Hamilton Laboratory Products, Reno, NV, USA). A $\beta$ solution ( $5 \mu \mathrm{L}$ ) was gradually injected into the region on the right side at a rate of $0.5 \mu \mathrm{L} / \mathrm{min}$. Then the syringe was left in place for 5 min after the injection before being dislodged to allow diffusion of $A \beta$. The next scalp was stitched. Also, Sham group rats operated like this surgical protocol with the same injection volume, but they received PBS (vehicle solution) instead of A $\beta$. Animals are allowed to have one week of recovery after surgery before beginning treatment (37).

\subsection{Preparation and gavage of Ecdy}

The dry powder of Ecdysterone supplementation (purchased from Amazon company, USA) dissolved in distilled water at a concentration of $10 \mathrm{mg} / \mathrm{cc}$ and then gavaged orally with an insulin syringe accompanied by a gavage needle in the amount of $10 \mathrm{mg} / \mathrm{kg}$. This protocol was carried out every day for eight weeks (33).

\subsection{HIIT exercise protocol}

One week after injection of $A \beta$, the exercise group rats were trained on a motorized rodent treadmill apparatus (Tajhiz Gostare Omide Iranian, Iran) (38). All rats were initially familiarized with the treadmill environment through walking at a speed between 5 to $10 \mathrm{~m} / \mathrm{min}$ for 10 minutes 5 days. After animals adapted to the environment, Rats ran on a treadmill with a $15^{\circ}$ inclination until exhaustion to measure their maximal oxygen uptake $\left(\mathrm{Vo}_{2}\right.$ max $)$. They started the protocol at speed of $6 \mathrm{~m} / \mathrm{min}$ and increased by $3 \mathrm{~m} / \mathrm{min}$ every $3 \mathrm{~min}$ until rats were unable to run (39). After the determination of $\mathrm{Vo}_{2}$ max, the main period of exercise began. The animals every day ran $1 \mathrm{~min}$ intervals at $90 \%$ of maximal exercise capacity, followed by $1 \mathrm{~min}$ intervals at $50 \%$ of maximal exercise capacity alternately at no incline ( $0 \%$ for 30 minutes in the first week and every week, 5 minutes added to the time of exercise until the time reached to 60 minutes in the seventh week (39). This protocol lasted for eight weeks. Besides, the main period of exercise included a $5 \mathrm{~min}$ warm-up and a $5 \mathrm{~min}$ cool-down at $40 \% \mathrm{VO}_{2} \mathrm{max}$ before and after the exercise period (40). At the same time, animals trained for five days over a week (they rested on Fridays and Mondays) between 9 AM and 2 PM.

\subsection{Morris Water Maze (MWM)}

\subsubsection{Morris water maze apparatus}

Spital memory and cognitive performance were assayed using a five days Morris water maze (MWM) test. The water maze device ( $180 \mathrm{~cm}$ in diameter and $60 \mathrm{~cm}$ in height) was full of $25 \pm 1{ }^{\circ} \mathrm{C}$ water to a depth of $45 \mathrm{~cm}$ located in a room containing a variety of visual cues that was allocated into four equal 
quadrants. In addition, an invisible circular platform (10 cm diameter) was submerged in a stable position at $1.5 \mathrm{~cm}$ beneath the water's surface in the center of the Northwest quadrant of the pool. Low lights were used for illumination, and the room was sound insulated. On the other hand, the pool was separated into four specific start points as the East $(E)$, West $(W)$, North $(N)$, and South $(S)$. These adjustments remained consistent for all rats across the training trials (41).

\subsubsection{Habituation}

Before starting the first training session, rats were put in the Morris water maze pool to swim without any platform for habituation to the environment for 60 seconds.

\subsubsection{Hidden platform training}

The training sessions were performed between $9 \mathrm{AM}$ and $12 \mathrm{PM}$ for four days, including. Eight trials were divided equally into two blocks (every block was four trials), that there was a five-minute break between every block for every rat. For each trial, the animals in all groups were placed in the pool (facing the pool wall) to start the trial at one of the four starting points in a different order in every trial and allowed to swim for 60 seconds from a start point $(E, W, N$, and $S)$ in the pool to find the invisible platform. If animals could detect the platform in 60 seconds, they were let to remain on the platform to rest and discover the environment for 30 seconds, but if they failed to find it, they were guided to the invisible platform by a technician and then were let to rest and discovery for 30 seconds. A video camera (Nikon, Melville, NY) linked to a tracking system in the computer was directly installed above the pool to record specific parameters, such as Distance moved by swimming and time spent to reach the platform (Scape latency) from the training sessions (41).

\subsubsection{Probe test}

On the fifth day, a probe test was conducted to evaluate spatial memory retention twenty-four hours after the training phase. On this day, the platform was eliminated, and each rat was placed in the pool like the training method and was allowed freely to swim for 60 seconds. Then, as an assay of spatial memory retention, the number of entrances to the target quadrant, time spent in the target quadrant, distance traveled in the target quadrant, and Average Speed was analyzed (42).

\subsection{5. visual test}

Forty-five minutes after the probe test, the platform elevated above the water surface and also was made apparent by a piece of bright sponge and located in the SE quadrant, and rats were let to swim and explore the visible platform for 60 seconds in order to evince their visual ability (43).

\subsection{Passive Avoidance Task (PAT)}

\subsubsection{Passive avoidance apparatus}


In the survey, a PAL device and a procedure (step-through method) were identical to the previous studies used to evaluate passive avoidance memory and learning (44-46). The device contained a bright cubical space with a dimension of (22_22_32 cm3) made of limpid plastic and a dark cubical space with dark opaque plastic walls $\left(22 \_22 \_32 \mathrm{~cm}^{3}\right)$. Also, the space floors were made of stainless steel shafts $(3 \mathrm{~mm}$ in diameter), placed at a 1-cm distance from each other. Besides, a shock generator was used for electrifying the dark chamber floor, and a rectangular opening $\left(6 \_8 \mathrm{~cm}^{2}\right)$ was placed between the two bright and dark spaces, which could be closed by an opaque guillotine door (41).

\subsubsection{Passive avoidance training}

In order to adapt the rats to the device, they were given two trials opportunity to adapt. At first, the rats entered into the bright section of the apparatus; then, after 30 seconds, the guillotine door picked up. With due attention to the natural trend of rats to the dark environment, they tried to enter the dark compartment (entrance defined as all the body entering the dark compartment). The guillotine door closed as the rats entered the dark compartment. After spending 30 seconds, they were deleted from the dark compartment and placed in their cages; the second trial repeated after $30 \mathrm{~min}$ precisely in the same way, and the experiment was pursued after the same pause by the first acquisition trial. In the acquisition trial, the guillotine door was picked up immediately after locating rats in the bright compartment, the entrance delay to the dark compartment or step-through latency to acquisition (STLa) was evaluated, and after entering rat to the dark compartment, the door closed, an electrical shock used ( $0.4 \mathrm{~mA})$ for 1.5 seconds. Then the rats were returned to a separate cage after 30 seconds; this protocol repeated for two more minutes after 120 seconds. Whenever the rats reentered in the dark compartment, they have received a foot shock, and the protocol was repeated, but by remaining them in the light compartment for all two minutes continuously, training interrupted, and the number of trials was recorded as the number of acquisitions. Also, the step-through latency in the acquisition trial (STLa) was recorded (41).

\subsubsection{Retention test}

Long-term memory as a retention trial 24 hours after the PAL acquisition trial was carried out. Like the PAL training session, the animals took in the light compartment and immediately opened the door. Then, the step-through latency in the retention trial (STLr) and the time spent in the dark compartment (TDC) were recorded for 10 minutes. Through the retention test, there were no electrical shocks. If animals did not enter the dark compartment within 300 seconds, the retention test terminated, and a ceiling score of 300 seconds was recorded (43).

\subsection{Nissl staining protocol}

A day after experiments finished, three rats in every group chose and were deeply anesthetized by ketamine $(100 \mathrm{mg} / \mathrm{kg})$ and xylazine $(10 \mathrm{mg} / \mathrm{kg})$ and perfused at first with normal saline and then $10 \%$ formalin for fixation through the heart and post-fixed in the same solution. After some months, formalinfixed brain samples were placed in a tissue processor in a 21 hours protocol and washed with tap water. Ethanol dilution (70\%,80\%,90\%, and $99 \%$ respectively) was used for dehydration, cleared in xylene, 
embedded in paraffin wax $\left(60^{\circ} \mathrm{C}\right)$ till being cold, and next sectioned with a microtome (Leitz $\mathrm{GmBH}$, Wetzlar, Germany) to obtain five $\mu \mathrm{m}$ thickness section and finally collected on glass slides. In staining protocol, brain sample slides deparaffinized in xylene (20 minutes), rehydrated in descending alcohol $(96 \%, 90 \%, 80 \%$, and $70 \%, 5$ minutes each solution), washed in tap water, stained with cresyl violet solution for 5 minutes, rewashed in tap water and destained in ascending alcohol solutions $(70 \%, 80 \%$, $90 \%$, and $96 \%$, (just washing)), and finally cleared in xylene. The number of intact hippocampal CA1 pyramidal cells in $1 \mathrm{~mm}$ length counted.

\subsection{Assay of hippocampus biochemical parameters}

At the end of the study, after behavioral tests, five rats in every group chose and were deeply anesthetized by ketamine $(100 \mathrm{mg} / \mathrm{kg})$ and xylazine $(10 \mathrm{mg} / \mathrm{kg})$ and brain them extracted from the skull, then hippocampus separated and froze at $-80^{\circ} \mathrm{C}$ after washing in PBS. Next, samples were sent for biochemistry measurements. Finally, hippocampus measurements were performed for Super Oxide Dismutase (SOD), Catalase (CAT), Glutathione Peroxidase (GPx) and Glutathione Reductase (GRx) as antioxidant enzymes concerning the current protocols (47-49).

\subsection{Statistical analysis}

Data expressed as mean \pm standard error of the mean (SEM) and The Graph Pad Prism version 8.0 (Graph Pad Software, San Diego, CA, USA) applied for statistical analysis. All data were analyzed by twoway and one-way analyses of variance (ANOVA) followed by Tukey's post hoc test. Results considered significantly different if $\mathrm{P}<0.05$.

\section{Results}

There was no significant difference between the control group, sham group, and positive control groups (Ecdy, HIIT, Ecdy+HIIT) $(P>0.05)$. Thus, in this survey, sham and positive control groups were ignored, and the Alzheimer group also treatment groups were just assessed with the control group.

\subsection{Effect of HIIT and Ecdy on body weights}

Bodyweight in all groups was controlled throughout the study. Differences between the Initial weights and final weights of groups are shown in (Fig.3). At the beginning of the survey, all groups were at the same weight; after approximately 12 weeks of the experiment, there was no significant difference between groups $\left(F_{8,63}=2.712 ; P=0.9860\right)$.

\subsection{Effect of HIIT and Ecdy treatment on the Marris water maze Test}

The results of two-way ANOVA with repeated measures indicated the statistically significant effect of days of training $\left(F_{3,165}=127.2 ; P<0.001\right)$ and treatment $\left(F_{8,63}=3.491 ; P=0.0021\right)$ in swimming distance in four days of training. Besides, swimming distance by rats in the Alzheimer group on the second $(P=0.0341)$, third $(P<0.001)$, and fourth day $(P=0.007)$ of the training days significantly climbed 
in comparison to the control group. On the other hand, the Ecdy treatment group on the third $(P=0.0411)$ and fourth day $(P=0.0232)$ and the treatment group of Ecdy+HIIT on the third $(P<0.001)$ and fourth day $(P=0.0034)$ significantly decreased it (Fig.4B).

Also, the results of two-way repeated measures ANOVA showed that the escape latency to the hidden platform in the Alzheimer group compared to the control group in the third $(P=0.0033)$ and forth $(P<$ $0.001)$ days of training significantly increased [treatment factor $\left(F_{8,63}=3.121 ; P=0.0049\right)$, day factor $\left.\left(F_{3,157}=120 ; P<0.001\right)\right]$. While, Ecdy (fourth day: $\left.P=0.0253\right)$ and Ecdy+HIIT treatment groups (third day: $P=0.0019$, fourth day: $P=0.0014$ ) decreased it significantly (Fig.4C).

However, the one-way ANOVA results showed no significant difference between Alzheimer's and control groups $\left(F_{8,63}=0.9980 ; P=0.4467\right)$ in the percentage of target quadrant entry in probe day. Also, no treatment group had a noticeable difference compared with the Alzheimer group ( $P>0.05)$ (Fig.4D).

In another valuable, One-way ANOVA repeated measures illustrated that there was a significant difference between Alzheimer and control groups $\left(F_{8,63}=1.757 ; P=0.0054\right)$ also, a significant difference between the Ecdy treatment group $(P=0.0340)$ and $E c d y+H I I T$ treatment group $(P=0.0392)$ with the Alzheimer group in the swimming distance in the target quadrant in probe day (Fig.4E).

Another data that was measured from the Morris water maze was the time spent in the target quadrant. Results showed the time that rats in the Alzheimer group spent in the target quadrant dropped significantly $\left(F_{8,63}=5.008 ; P<0.001\right)$, although the Ecdy+HIIT treatment group caused an increase in it noticeably $(P=0.0026)($ Fig.4F).

Likewise, the one-way ANOVA Tukey test indicated that there was not any significant difference in the average speed in probe trial between Alzheimer and control groups $\left(F_{8,63}=1.533 ; P=0.5741\right)$, also among treatment and Alzheimer groups $(P>0.05)$ (Fig.4G).

\subsection{Effect of HIIT and Ecdy treatment on the Passive Avoidance Task}

Concerning one-way ANOVA results, there was no significant difference among initial latency or stepthrough latency in acquisition trial $\left(F_{8,63}=0.7217 ; \mathrm{P}>0.05\right)$ (Fig.5A).

In addition, one-way ANOVA analysis illustrates that step-through latency in the retention test in the passive avoidance task decreased in the Alzheimer group compared with the control group $\left(F_{8,63}=37 ; \mathrm{P}\right.$ $<0.001)$. On the other side, Ecdy $(P=0.0251)$ and Ecdy+HIIT $(P<0.001)$ treatment groups increased noticeably compared to the Alzheimer group (Fig.5A).

Moreover, counting time in the dark compartment by one-way ANOVA showed a significant difference between Alzheimer's and control groups $\left(F_{8,63}=47.96 ; P<0.001\right)$. Moreover, there were significant differences between Ecdy treatment and Alzheimer group $(P=0.0132)$ also, Ecdy+HIIT treatment and Alzheimer group $(P<0.001)$ (Fig.5B). 
Also, concerning the results, it was clear that there were not any significant differences between groups in the number of trials to acquisition in the passive avoidance task $\left(F_{8,63}=2.681 ; \mathrm{P}>0.05\right)$ (Fig.5C).

3.4. Effect of HIIT and Ecdy treatment on the histological changes in the cerebral cortex and hippocampus

Based on the results shown in Fig. 6, histological changes in the hippocampal CA1, CA3 and DG areas and also cerebral cortex measured by Nissl staining. A significant decrease found in the number of intact neurons of the hippocampal CA1 $\left(F_{4,10}=16.88 ; P<0.001\right)$, CA3 $\left(F_{4,10}=49.25 ; P<0.001\right)$, and DG $\left(F_{4,10}=\right.$ 44.42; $P<0.001)$ areas and $C C\left(F_{4,10}=6.308 ; P=0.0072\right)$ of the Alzheimer group compared to the Control groups. While, Ecdy treatment group could increase number of intact neurons significantly in hippocampal CA1, CA3, DG areas of the brain compare to the Alzheimer group $(P=0.0205 ; P=0.0171 ; P$ $=0.0186$ respectively). Meanwhile, Ecdy+HIIT treated group markedly culminated in improvement of neuronal distraction in the hippocampal CA1 $(P=0.0022), C A 3(P<0.001), D G(P<0.001)$ areas and also in $C C(P=0.0407)$ compared with the Alzheimer group (Fig.6).

\subsection{Effect of HIIT and Ecdy treatment on hippocampus oxidative stress parameters}

ANOVA one-way repeated measures indicated Superoxide Dismutase (SOD) that is known as an antioxidant enzyme and prevents oxidative stress using superoxide ion $\left(\mathrm{O}_{2}{ }^{-}\right)$neutralization in the Alzheimer group dropped significantly $\left(F_{8,36}=5.173 ; P<0.001\right)$ and treatment groups of Ecdy and HIIT+Ecdy increased it noticeably near to the normal range $(P=0.0288, P=0.0039$ respectively) (Fig.7A).

On the other hand, results revealed that CAT, as one of the crucial enzymes to neutralize Hydrogen peroxide $\left(\mathrm{H}_{2} \mathrm{O}_{2}\right)$, decreased significantly in the Alzheimer group compare to the control group $\left(\mathrm{F}_{8,36}=\right.$ 8.815; $\mathrm{P}<0.001)$. In comparison, Ecdy and group of HIIT+Ecdy could promote the amount of SOD considerably in the Alzheimer group $(P=0.0276, P<0.001$ respectively) (Fig.7B).

Besides, Glutathione Peroxidase (GPx), that is a helpful enzyme in avoiding stress oxidative notably decreased in the Alzheimer group compare to the control group $\left(F_{8,36}=6.753 ; P=0.0059\right)$. In comparison, HIIT+Ecdy could refine the amount of GPx significantly in the Alzheimer group $(P=0.0199)$ (Fig.7C).

Also, results showed Glutathione Reductase (GRx), as an antioxidant enzyme that converts GSSG to GSH, declined significantly in the Alzheimer group compare to the control group $\left(F_{8,36}=18.89 ; P=0.0015\right)$. In comparison, Ecdy and group of HIIT+Ecdy could promote the amount of SOD considerably in the Alzheimer group $(P=0.0019, P=0.0079$ respectively) (Fig.7D).

\section{Discussion}

In the present survey, $A \beta$-induced experimental Alzheimer produced a wide array of disorders such as spatial memory impairments in MWM and decreased learning and memory in PAT. These behavioral 
inadequacies were related to the $A \beta$ concentration in the hippocampus and cerebral cortex and oxidative stress that is one of the fundamental causes for $A \beta$-induced tissue damage. Under such a condition, extra amounts of reactive oxygen species (ROS) lead to oxidative damage, including lipid peroxidation and protein oxidation, culminating in programmed cell death and neuronal loss in the hippocampus and cerebral cortex.

Conversely, eight weeks HIIT as a new training method did not alter any behavioral alteration separately in the Morris water maze in the $A \beta$-induced Alzheimer rats. While Ecdy as a natural hormone decreased swimming distance and escape latency to the hidden platform in training days, it also caused an improvement in total swimming distance in the probe day. Additionally, eight weeks of HIIT accompanied by Ecdy could help decrease swimming distance and escape latency to the hidden platform in training days, and also, they caused an improvement in total swimming distance and total time spent in the target quadrant in the probe day. Besides, it was specified that eight weeks of performing HIIT did not alleviate learning and memory impairments as a result of $A \beta$ concentration in the hippocampus in none of the valuables in the passive avoidance task. Nevertheless, eight weeks of consumption of Ecdy and a combination of both HIIT and Ecdy could promote latency time to the dark compartment (STLr) and time spent in the dark compartment (TDC). Histological studies through Nissl staining demonstrated that eight weeks of Ecdy separately increased the number of intact neurons in the hippocampal DG, CA1, CA3 areas but not CC. In addition, although HIIT exercise separately did not have any impact on the neuronal population, a combination of eight weeks consumption of Ecdy accompanied with eight weeks HIIT culminated in an increase in intact neurons of the hippocampal DG, CA1, CA3 hippocampus areas, and $C C$ that were declined as a result of $A \beta$ injection. Also, biomechanical experiments revealed that consumption of Ecdy separate (except GPx) and in combination with HIIT exercise augmented to the Superoxide Dismutase (SOD), Catalase (CAT), Glutathione Peroxidase (GPx) and Glutathione Reductase (GRx) enzymes in the hippocampus tissue that were dropped because of $A \beta$ injection. In contrary, HIIT exercise did not alter content of antioxidant enzymes in the hippocampus after subtraction due to $A \beta$ injection.

Furthermore, previous studies have reported the beneficial effects of Ecdy and HIIT alone in advancing learning and memory dysfunctions. A study indicated that HIIT for eight minutes per day promotes memory and learning impairment in $A \beta$-induced rats; it was due to an increase in antioxidant enzymes of Super Oxide Dismutase and Catalase and decreased Lipid peroxidation in the hippocampus. Also, the study showed gene expression levels of BDNF that were dropped because of $A$ injection after HIIT exercise improved (50). The other survey reported that six weeks of HIIT exercise (6 sessions per week) caused a significant increase in Super Oxide Dismutase antioxidant enzyme and Total Antioxidant Capacity in the cerebellum but not in the cerebral cortex. In addition, in this study, there was a significant difference between anxiety behaviors and recognition memory before and after HIIT exercise (51). A study conducted on traumatic brain injury illustrated HIIT caused an improvement in recognition performance in novel object recognition tests and anxiety behaviors in the elevated plus-maze that were impaired as a to culminate of trauma. Moreover, after biochemical assays were declared trauma increased MDA content and decreased Super Oxid Dismutase, Catalase, and glutathione in the 
hippocampus, treatment with HIIT could alleviate all the above factors near the normal range (52). Furthermore, according to another survey, Parkinson's disease demolished short-term memory in the $Y$ maze test and decreased gene expression levels of BDNF in the hippocampus, but HIIT exercise treated short-term memory and increased gene expression levels of BDNF (53).

On the other side, a survey showed that a particular dosage of Ecdy promoted spatial memory and learning in the rats that faced a memory impairment after $A \beta_{(35-25)}$ injection. Also, after $A \beta_{(32-25)}$ injection, the gene expression level of $\mathrm{C}$-fos is an indicator for neuronal activity in the brain and has a close relation with memory and learning decreased but, consumption of Ecdy increased it near to the average amount (54). The study with Shihao et al. on traumatic brain injury (TBI) in rats reported that peritoneal injection of Ecdy $(16 \mathrm{mg} / \mathrm{kg})$ for seven days resulted in significant decreases in the neuronal death rate, brain water content, MDA content, and a noticeable increase in SOD enzymes in the hippocampus compared with TBI group that illustrated therapeutic effects of Ecdy on TBI through inhibiting free radical damage and brain edema (55). According to XichaoXia et al., type 1 diabetes damaged the hippocampus CA1 area, caused higher expression levels of NF-kB as an inflammation factor, and exhibited significant memory loss. Moreover, the expression levels of Super Oxide Dismutase, catalase, GSH-Px Glutathione Reductase, and BDNF were significantly decreased in the diabetic rats. Despite this, the treatment with Ecdy in three dosages $(1,10,100 \mathrm{mg} / \mathrm{kg} /$ day), especially in higher dosage for 12 weeks, orally reversed the conditions mentioned above caused by diabetes (33).

In addition, never should it be forgotten that this survey is the first research in which the protective effects of HIIT and Ecdy next to each other on memory deficits, stress oxidative enzymes, and neuronal loss in the rat models of Alzheimer's disease are investigated.

\section{Conclusion}

The present survey results (Fig.8) displayed that, although Ecdysterone has antioxidant properties separately and could help alleviate neuronal loss and behavioral deficits a little bit, a treatment included combination of Ecdysterone with HIIT exercise has a substantial antioxidant effect on A $\beta$-induced tissues. Hence, a combination of both causes synaptic refinement and behavioral improvement concerning the prepared study. However, further and more fundamental researches are required to investigate the involved mechanisms in detail.

\section{Declarations}

\section{Statements and declarations}

Parsa Gholipour: Conceptualization, Writing - review \& editing, Data curation, Visualization, Project administration, Funding acquisition, Formal analysis. Alireza Komaki: Methodology, Writing - review \& editing. Mahdi Ramezani: Supervision, Conceptualization, Writing - review \& editing, Data curation, Visualization, Project administration, Funding acquisition, Formal analysis. 
The authors declare that they have no known competing financial interests or personal relationships that could have appeared to influence the work reported in this paper.

\section{Acknowledgments}

This research was supported by a Grant from the Hamadan University of Medical Sciences, Hamadan, Iran. The authors would like to express their gratitude to the staff of the Neurophysiology Research Center for helping them carry out this project.

\section{Data Availability Statement}

The raw data supporting the conclusions of this article will be made available by the authors, without undue reservation, to any qualified researcher.

\section{References}

1. Lee YK, Choi IS, Ban JO, Lee HJ, Lee US, Han SB, et al. 4-0-methylhonokiol attenuated $\beta$-amyloidinduced memory impairment through reduction of oxidative damages via inactivation of p38 MAP kinase. The Journal of nutritional biochemistry. 2011;22(5):476-86.

2. D Skaper S, Facci L, Zusso M, Giusti P. Synaptic plasticity, dementia and Alzheimer disease. CNS \& Neurological Disorders-Drug Targets (Formerly Current Drug Targets-CNS \& Neurological Disorders). 2017;16(3):220-33.

3. McGeer PL, McGeer EG. The amyloid cascade-inflammatory hypothesis of Alzheimer disease: implications for therapy. Acta neuropathologica. 2013;126(4):479-97.

4. Liao S, Deng H, Huang S, Yang J, Wang S, Yin B, et al. Design, synthesis and evaluation of novel 5, 6, 7-trimethoxyflavone-6-chlorotacrine hybrids as potential multifunctional agents for the treatment of Alzheimer's disease. Bioorganic \& medicinal chemistry letters. 2015;25(7):1541-5.

5. Salgado-Puga K, Pena-Ortega F. Cellular and network mechanisms underlying memory impairment induced by amyloid $\beta$ protein. Protein and peptide letters. 2015;22(4):303-21.

6. E Abdel Moneim A. Oxidant/antioxidant imbalance and the risk of Alzheimer's disease. Current Alzheimer Research. 2015;12(4):335-49.

7. Fotuhi M, Do D, Jack C. Modifiable factors that alter the size of the hippocampus with ageing. Nature Reviews Neurology. 2012;8(4):189-202.

8. Alosco ML, Brickman AM, Spitznagel MB, Griffith EY, Narkhede A, Raz N, et al. The adverse impact of type 2 diabetes on brain volume in heart failure. Journal of clinical and experimental neuropsychology. 2013;35(3):309-18.

9. Jiang T, Sun Q, Chen S. Oxidative stress: a major pathogenesis and potential therapeutic target of antioxidative agents in Parkinson's disease and Alzheimer's disease. Progress in neurobiology. 2016;147:1-19. 
10. Arab H, Mahjoub S, Hajian-Tilaki K, Moghadasi M. The effect of green tea consumption on oxidative stress markers and cognitive function in patients with Alzheimer's disease: A prospective intervention study. Caspian journal of internal medicine. 2016;7(3):188.

11. Parveen A, Akash MSH, Rehman K, Kyunn WW. Recent investigations for discovery of natural antioxidants: a comprehensive review. Critical Reviews ${ }^{\mathrm{TM}}$ in Eukaryotic Gene Expression. 2016;26(2).

12. A Sobenin I, A Myasoedova V, N Orekhov A. Phytoestrogen-rich dietary supplements in antiatherosclerotic therapy in postmenopausal women. Current pharmaceutical design. 2016;22(2):15263.

13. Tarrant AM, Reitzel AM, Blomquist $\mathrm{CH}$, Haller F, Tokarz J, Adamski J. Steroid metabolism in cnidarians: insights from Nematostella vectensis. Molecular and cellular endocrinology. 2009;301(12):27-36.

14. Jian C-X, Liu X-F, Hu J, Li C-J, Zhang G, Li Y, et al. 20-hydroxyecdysone-induced bone morphogenetic protein-2-dependent osteogenic differentiation through the ERK pathway in human periodontal ligament stem cells. European journal of pharmacology. 2013;698(1-3):48-56.

15. Hung T-J, Chen W-M, Liu S-F, Liao T-N, Lee T-C, Chuang L-Y, et al. 20-Hydroxyecdysone attenuates TGF- $\beta 1$-induced renal cellular fibrosis in proximal tubule cells. Journal of diabetes and its complications. 2012;26(6):463-9.

16. Tóth N, Szabó A, Kacsala P, Héger J, Zádor E. 20-Hydroxyecdysone increases fiber size in a musclespecific fashion in rat. Phytomedicine. 2008;15(9):691-8.

17. Lafont R, Dinan L. Practical uses for ecdysteroids in mammals including humans: and update. Journal of insect science. 2003;3(1).

18. Catalan R, Martinez A, Aragones M, Miguel B, Robles A, Godoy J. Alterations in rat lipid metabolism following ecdysterone treatment. Comparative biochemistry and physiology $B$, Comparative biochemistry. 1985;81(3):771-5.

19. Oehme I, Bösser S, Zörnig M. Agonists of an ecdysone-inducible mammalian expression system inhibit Fas Ligand-and TRAIL-induced apoptosis in the human colon carcinoma cell line RKO. Cell Death \& Differentiation. 2006;13(2):189-201.

20. Osinskaia L, Saad L, luD K. Antiradical properties and antioxidant activity of ecdysterone. Ukrainskii biokhimicheskii zhurnal (1978). 1992;64(1):114-7.

21. Su-fen Y, Zhong-jun W, Zheng-qin Y, Qin W, Qi-hai G, Qi-xin Z, et al. Protective effect of ecdysterone on PC12 cells cytotoxicity induced by Beta-amyloid 25-35. Chinese journal of integrative medicine. 2005;11(4):293-6.

22. Yang Z-Q, Wu Q, Lu Y-F, Yang S-F, Shi J-S, Li S-L. The Effect of ECR on the Learning and Memory

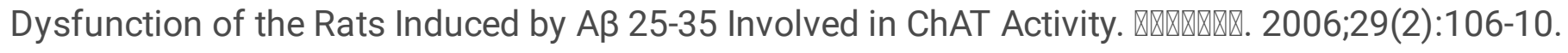

23. Li H, Liang A, Guan F, Fan R, Chi L, Yang B. Regular treadmill running improves spatial learning and memory performance in young mice through increased hippocampal neurogenesis and decreased stress. Brain research. 2013;1531:1-8. 
24. Liu YF, Chen Hi, Wu CL, Kuo YM, Yu L, Huang AM, et al. Differential effects of treadmill running and wheel running on spatial or aversive learning and memory: roles of amygdalar brain-derived neurotrophic factor and synaptotagmin I. The Journal of physiology. 2009;587(13):3221-31.

25. Dao AT, Zagaar MA, Levine AT, Salim S, Eriksen JL, Alkadhi KA. Treadmill exercise prevents learning and memory impairment in Alzheimer's disease-like pathology. Current Alzheimer Research. 2013;10(5):507-15.

26. Dao AT, Zagaar MA, Salim S, Eriksen JL, Alkadhi KA. Regular exercise prevents non-cognitive disturbances in a rat model of Alzheimer's disease. International Journal of Neuropsychopharmacology. 2014;17(4):593-602.

27. Afzalpour ME, Chadorneshin HT, Foadoddini M, Eivari HA. Comparing interval and continuous exercise training regimens on neurotrophic factors in rat brain. Physiology \& behavior. 2015;147:7883.

28. Freitas DA, Rocha-Vieira E, Soares BA, Nonato LF, Fonseca SR, Martins JB, et al. High intensity interval training modulates hippocampal oxidative stress, BDNF and inflammatory mediators in rats. Physiology \& behavior. 2018;184:6-11.

29. Li B, Liang F, Ding X, Yan Q, Zhao Y, Zhang X, et al. Interval and continuous exercise overcome memory deficits related to $\beta$-Amyloid accumulation through modulating mitochondrial dynamics. Behavioural brain research. 2019;376:112171.

30. Yan S-t, Gao F, Dong T-w, Fan H, Xi M-m, Miao F, et al. Meta-Analysis of Randomized Controlled Trials of Xueshuantong Injection in Prevention of Deep Venous Thrombosis of Lower Extremity after Orthopedic Surgery. Evidence-Based Complementary and Alternative Medicine. 2020;2020.

31. Lautenschlager NT, Cox KL, Flicker L, Foster JK, Van Bockxmeer FM, Xiao J, et al. Effect of physical activity on cognitive function in older adults at risk for Alzheimer disease: a randomized trial. Jama. 2008;300(9):1027-37.

32. Karamian R, Komaki A, Salehi I, Tahmasebi L, Komaki H, Shahidi S, et al. Vitamin C reverses leadinduced deficits in hippocampal synaptic plasticity in rats. Brain research bulletin. 2015;116:7-15.

33. Xia X, Zhang Q, Liu R, Wang Z, Tang N, Liu F, et al. Effects of 20-hydroxyecdysone on improving memory deficits in streptozotocin-induced type 1 diabetes mellitus in rat. European journal of pharmacology. 2014;740:45-52.

34. Pereira F, de Moraes R, Tibiriçá E, Nóbrega AC. Interval and continuous exercise training produce similar increases in skeletal muscle and left ventricle microvascular density in rats. BioMed research international. 2013;2013.

35. Zhang L-I, Sui H-j, Liang B, Wang H-m, Qu W-h, Yu S-x, et al. Atorvastatin prevents amyloid- $\beta$ peptide oligomer-induced synaptotoxicity and memory dysfunction in rats through a p38 MAPK-dependent pathway. Acta Pharmacologica Sinica. 2014;35(6):716-26.

36. Lorenzo A, Yankner BA. Beta-amyloid neurotoxicity requires fibril formation and is inhibited by congo red. Proceedings of the National Academy of Sciences. 1994;91(25):12243-7.

37. Paxinos G, Watson C. The rat brain in stereotaxic coordinates: hard cover edition: Elsevier; 2006. 
38. Mohammadkhani R, Khaledi N, Rajabi H, Salehi I, Komaki A. Influence of the maternal high-intensityinterval-training on the cardiac Sirt6 and lipid profile of the adult male offspring in rats. PloS one. 2020;15(8):e0237148.

39. Ferreira JC, Rolim NP, Bartholomeu JB, Gobatto CA, Kokubun E, Brum PC. Maximal lactate steady state in running mice: effect of exercise training. Clinical and Experimental Pharmacology and Physiology. 2007;34(8):760-5.

40. Lu K, Wang L, Wang C, Yang Y, Hu D, Ding R. Effects of high-intensity interval versus continuous moderate-intensity aerobic exercise on apoptosis, oxidative stress and metabolism of the infarcted myocardium in a rat model. Molecular medicine reports. 2015;12(2):2374-82.

41. Ramezani M, Komaki A, Hashemi-Firouzi N, Mortezaee K, Faraji N, Golipoor Z. Therapeutic effects of melatonin-treated bone marrow mesenchymal stem cells (BMSC) in a rat model of Alzheimer's disease. Journal of Chemical Neuroanatomy. 2020;108:101804.

42. Ghazvini H, Khaksari M, Esmaeilpour K, Shabani M, Asadi-Shekaari M, Khodamoradi M, et al. Effects of treatment with estrogen and progesterone on the methamphetamine-induced cognitive impairment in ovariectomized rats. Neuroscience letters. 2016;619:60-7.

43. Zarrinkalam E, Heidarianpour A, Salehi I, Ranjbar K, Komaki A. Effects of endurance, resistance, and concurrent exercise on learning and memory after morphine withdrawal in rats. Life sciences. 2016;157:19-24.

44. Hasanein P, Shahidi S. Effects of Hypericum perforatum extract on diabetes-induced learning and memory impairment in rats. Phytotherapy research. 2011;25(4):544-9.

45. Hasanein P, Shahidi S. Preventive effect of Teucrium polium on learning and memory deficits in diabetic rats. Medical science monitor: International medical journal of experimental and clinical research. 2012;18(1):BR41.

46. Komaki A, Karimi SA, Salehi I, Sarihi A, Shahidi S, Zarei M. The treatment combination of vitamins E and $\mathrm{C}$ and astaxanthin prevents high-fat diet induced memory deficits in rats. Pharmacology Biochemistry and Behavior. 2015;131:98-103.

47. Genet S, Kale RK, Baquer NZ. Alterations in antioxidant enzymes and oxidative damage in experimental diabetic rat tissues: effect of vanadate and fenugreek (Trigonella foenum graecum). Molecular and cellular biochemistry. 2002;236(1):7-12.

48. Rotruck J, Pope A, Ganther HE, Swanson A, Hafeman DG, Hoekstra W. Selenium: biochemical role as a component of glutathione peroxidase. Science. 1973;179(4073):588-90.

49. Pinto R, Bartley W. The effect of age and sex on glutathione reductase and glutathione peroxidase activities and on aerobic glutathione oxidation in rat liver homogenates. Biochemical Journal. 1969;112(1):109-15.

50. Vasconcelos-Filho FS, da Rocha-E-Silva RC, Martins JE, Godinho WD, da Costa VV, Ribeiro JK, et al. Neuroprotector Effect of Daily 8-Minutes of High-Intensity Interval Training in Rat A $31-42$ Alzheimer Disease Model. Current Alzheimer Research. 2020;17(14):1320-33. 
51. Freitas DA, Rocha-Vieira E, De Sousa RAL, Soares BA, Rocha-Gomes A, Garcia BCC, et al. Highintensity interval training improves cerebellar antioxidant capacity without affecting cognitive functions in rats. Behavioural brain research. 2019;376:112181.

52. Koyuncuoğlu T, Sevim H, Çetrez N, Meral Z, Gönenç B, Dertsiz EK, et al. High intensity interval training protects from Post Traumatic Stress Disorder induced cognitive impairment. Behavioural brain research. 2021;397:112923.

53. Sabaghi A, Heirani A, Mahmoodi H, Sabaghi S. High-intensity interval training prevents cognitivemotor impairment and serum BDNF level reduction in parkinson mice model. Sport Sciences for Health. 2019;15(3):681-7.

54. Yang S, Yang Z, Zhou Q, Wu Q, Huang X, Shi J. Effect of ecdysterone on the expression of c-fos in the brain of rats induced by microinjection beta-AP25-35 into the hippocampus. Yao xue xue bao= Acta pharmaceutica Sinica. 2004;39(4):241-4.

55. Shihao G, Zhengheng T, Tunan C, Fei L, You'an S, Hua F. Ecdysterone protects brain injury against free radical in rats. Journal of Third Military Medical University. 2012;2012:23.

\section{Figures}

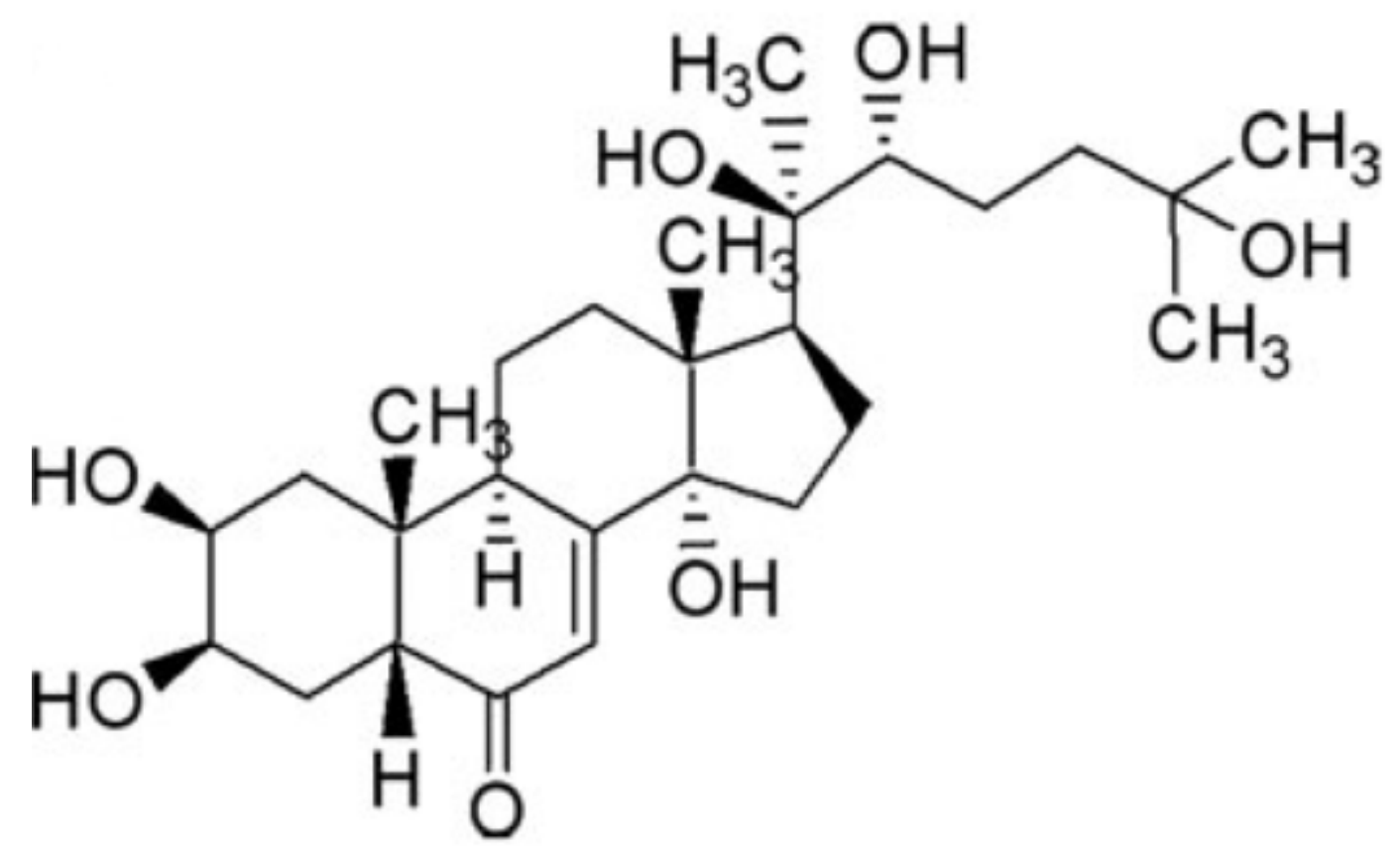

\section{Figure 1}

Chemical structures of Ecdy. 


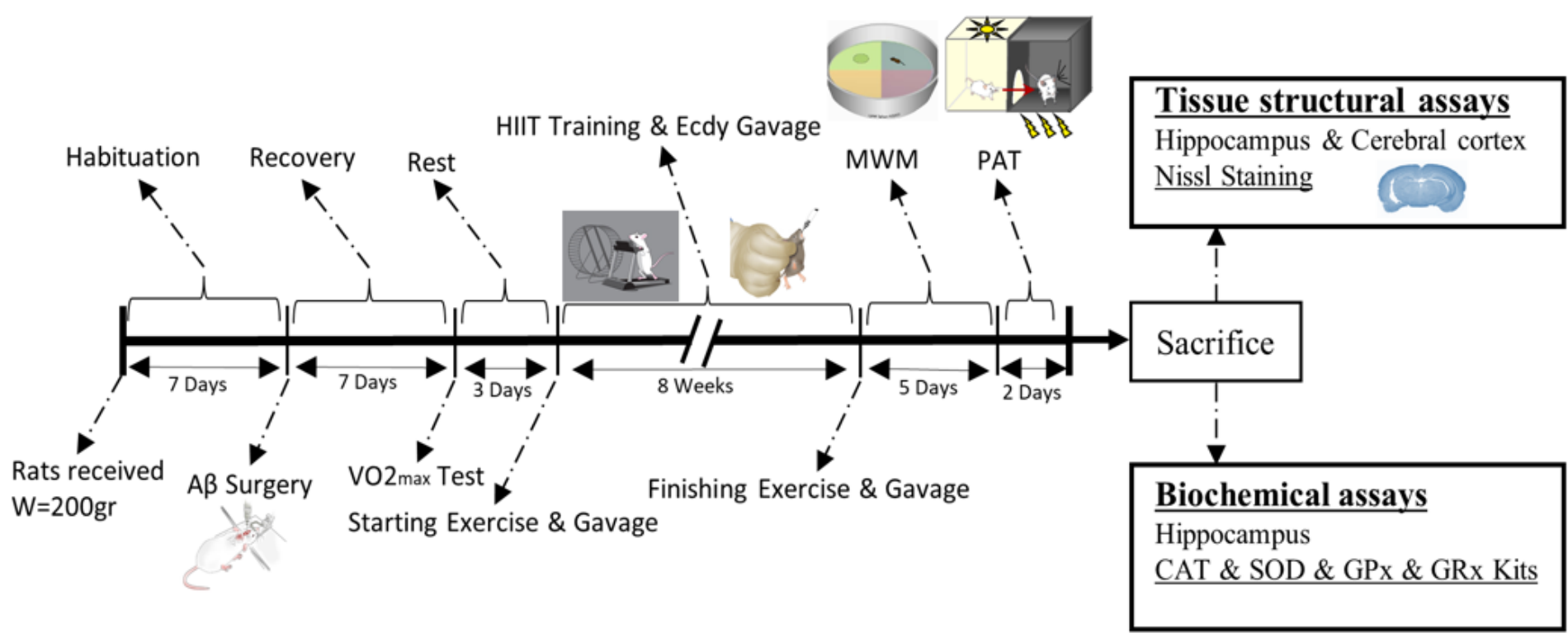

Figure 2

schematic of the experimental design and timeline. 


\section{Body Weight}

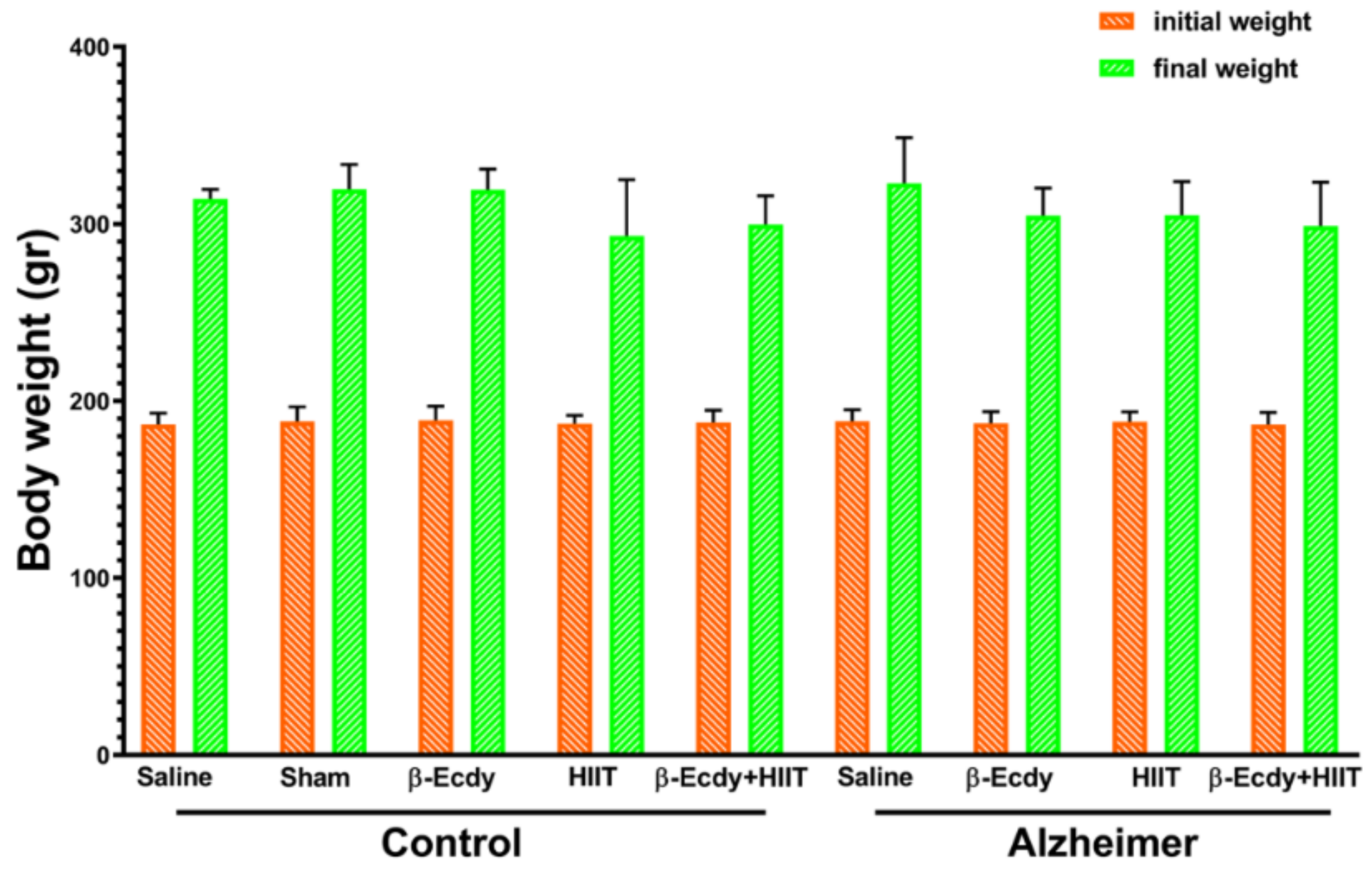

Figure 3

Initial weight and final weights. 


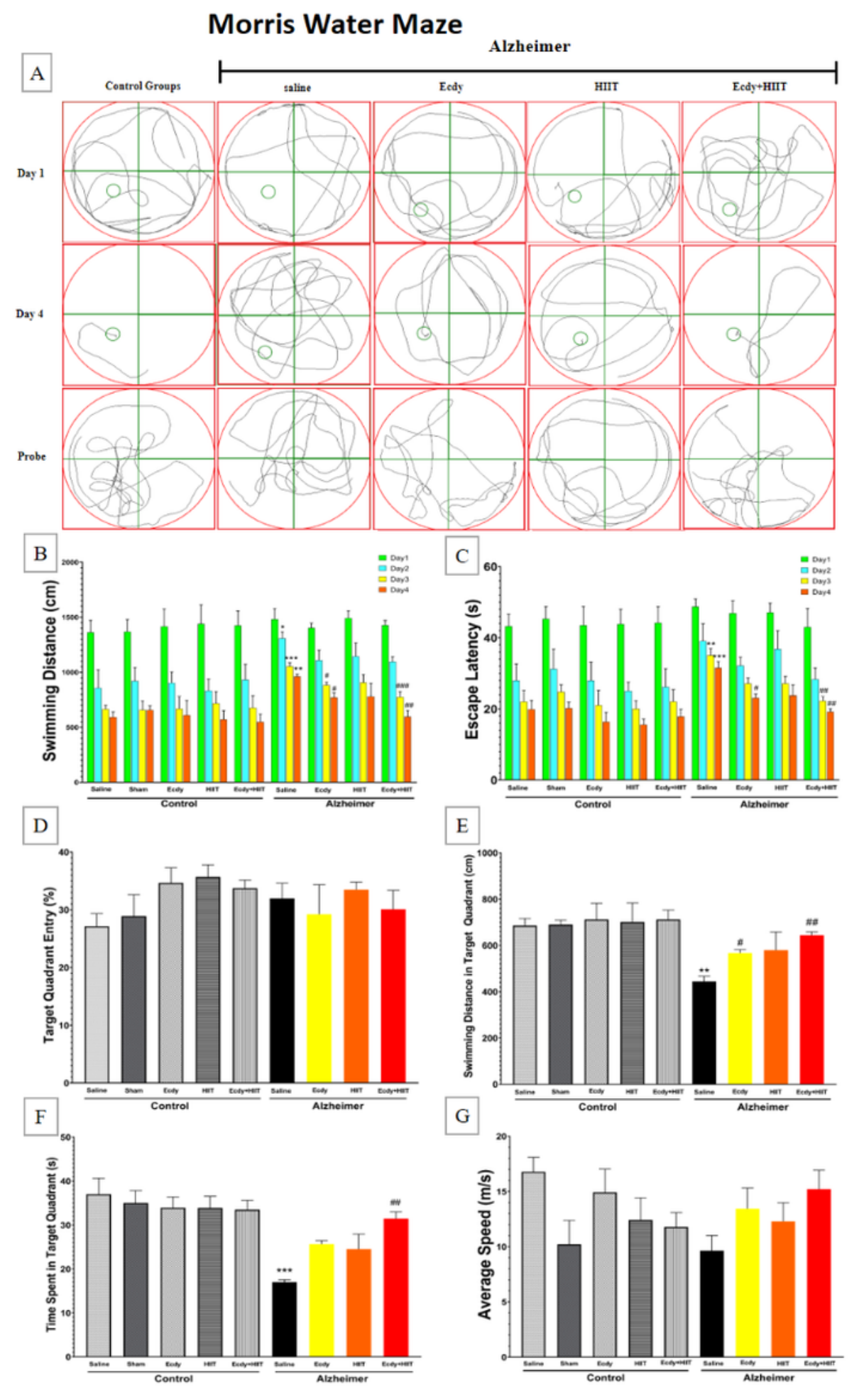

\section{Figure 4}

effect of HIIT and Ecdy treatment on swimming distance and escape latency to the hidden platform in the training trials and percentage of target quadrant entry, swimming distance in target quadrant, time spent in target quadrant and average speed in the probe trial in the Barnes Maze Test. Each bar represents

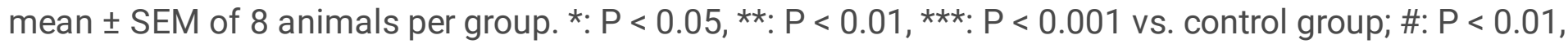
\#\#: $\mathrm{P}<0.01$, \#\#\#: $\mathrm{P}<0.001$ vs. the Alzheimer group. 
Passive Avoidance Task
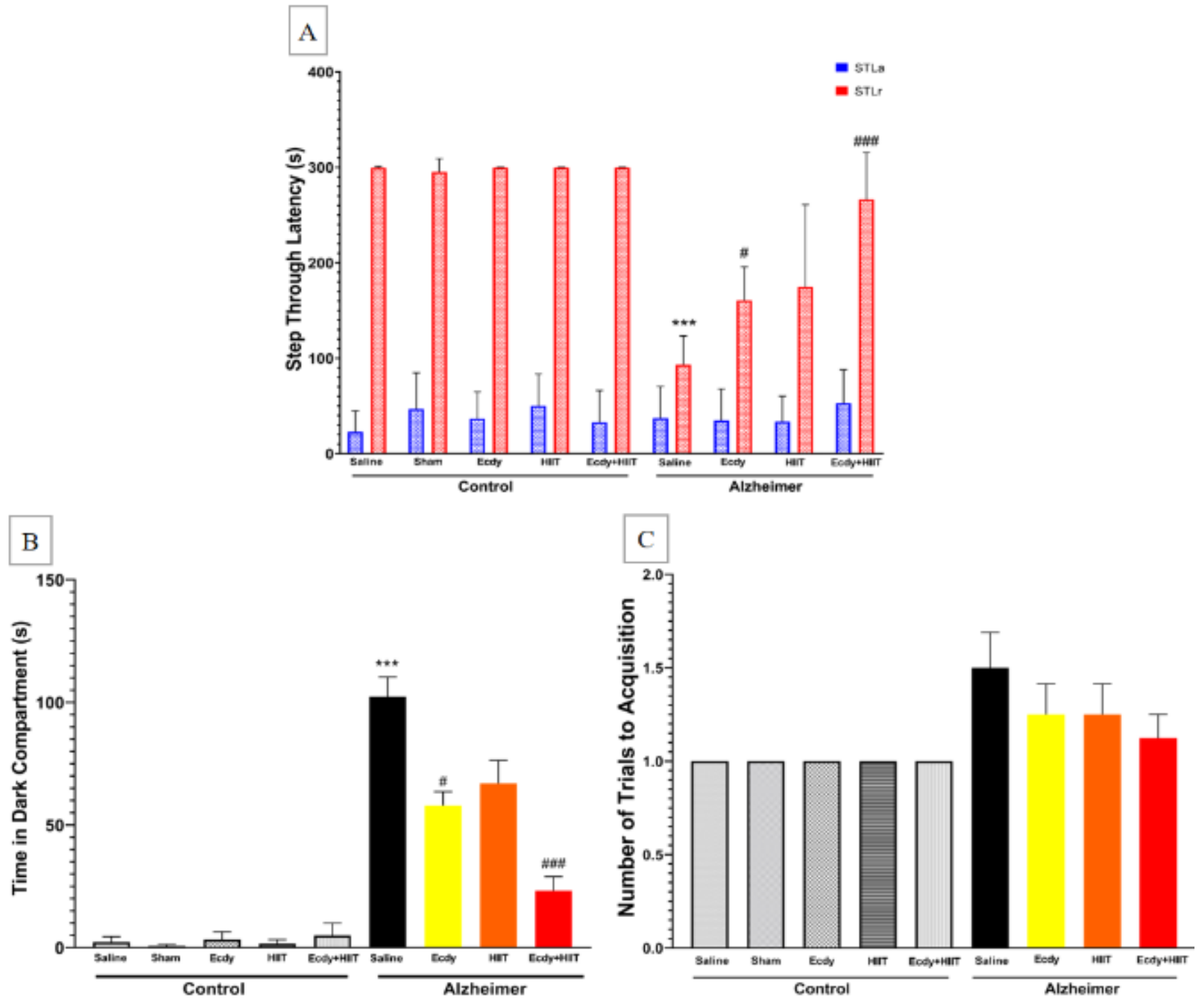

Figure 5

effect of HIIT and Ecdy treatment on the step through latency in acquisition trial and retention test separately, time in dark compartment and number of trials to acquisition in the passive avoidance task. Each bar represents mean \pm SEM of 8 animals per group. $* \star *: P<0.001$ vs. control group; \#: $P<0.01$, \#\#\#: $\mathrm{P}<0.001$ vs. the Alzheimer group. 


\section{Nissl Staining}

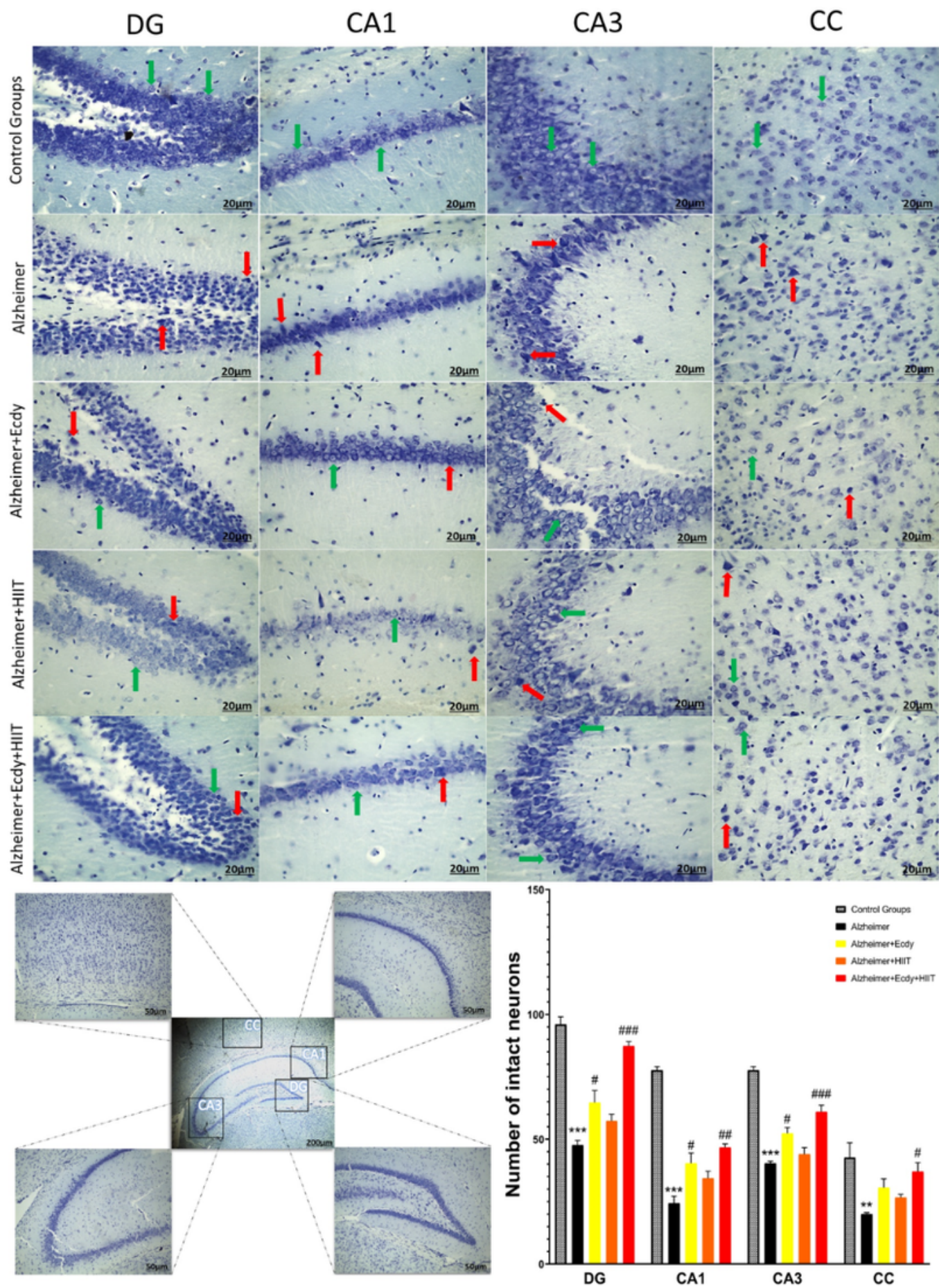

Figure 6

Effects of HIIT and Ecdy treatment on histological changes in the cerebral cortex (CC) and hippocampal CA1, CA3, and DG regions (cresyl violet (Nissl) stain, scale bar 200,50,20 $\mu \mathrm{m}$ ) of A 3 -induced rats. The 20 $\mu \mathrm{m}$ photographs in figure illustrates intact neurons (clear cells with distinct round nuclei, identified by green arrows) and dark neurons (shrinkage of cells with pyknotic nuclei, identified by red arrows), the 200 $\mu \mathrm{m}$ photographs represents photomicrograph of cerebral cortex and hippocampal regions of the rat (Nissl 
stain, 40xmagnification), also the figure includes the quantitative data of the number of intact neurons. Each bar represents mean \pm SEM of 3 animals per group. **: $P<0.01$ vs. control group; \#: $P<0.01$, \#\#: $P$ $<0.01$, \#\#\#: $\mathrm{P}<0.001$ vs. the Alzheimer group.

\section{Stress Oxidative Parameters}

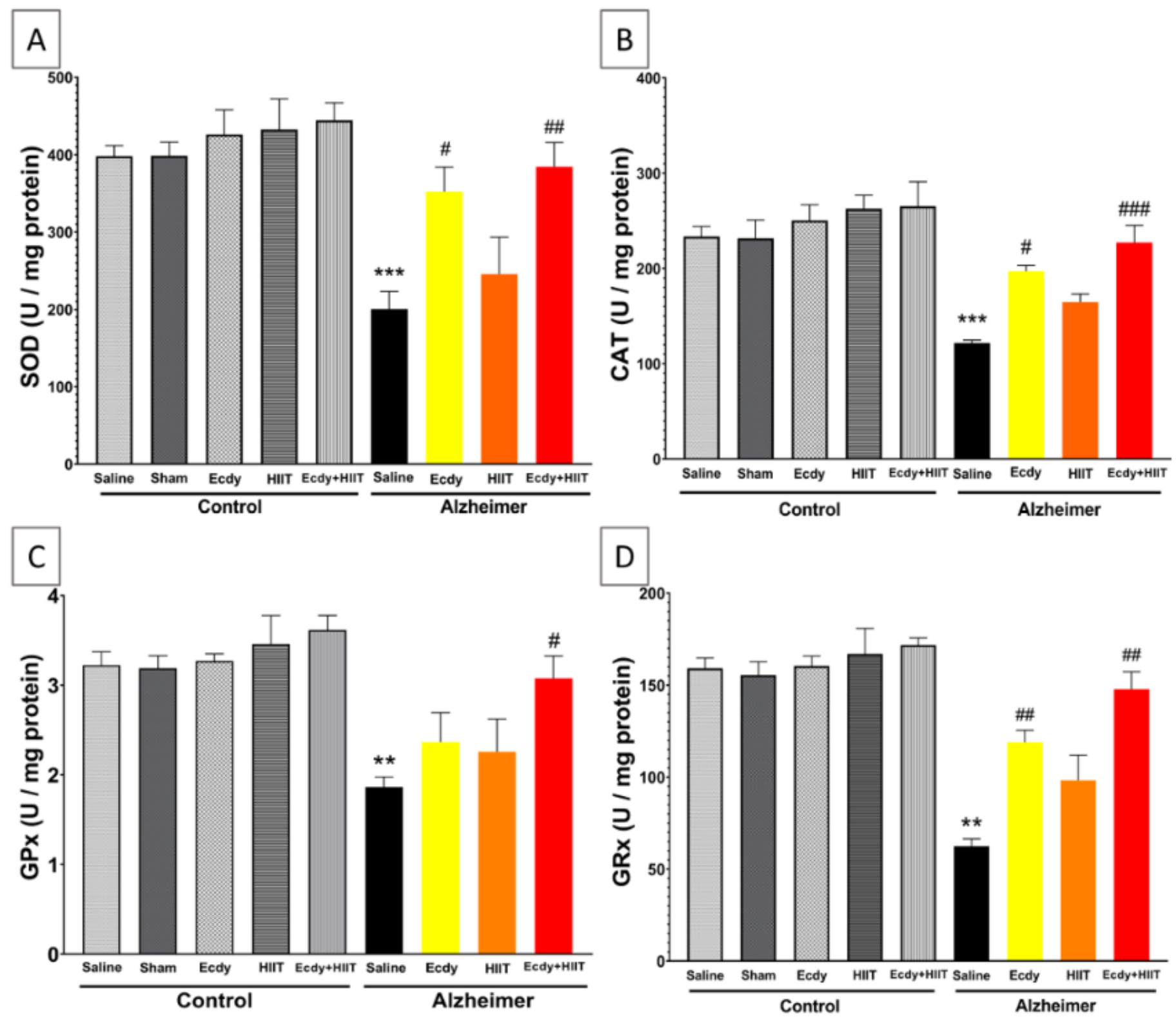

Figure 7

Effect of HIIT and Ecdy treatment on antioxidant enzymes of SOD, CAT, GPx and GRx of the hippocampus of the $A \beta$-induced rats. Each bar represents mean \pm SEM of 5 animals per group. ${ }^{\star *}$ : $P<0.01$, $* \star *: P<$ 0.001 vs. control group; \#: $P<0.01$, \#\#: $P<0.01$, \#\#: $P<0.001$ vs. the Alzheimer group. 


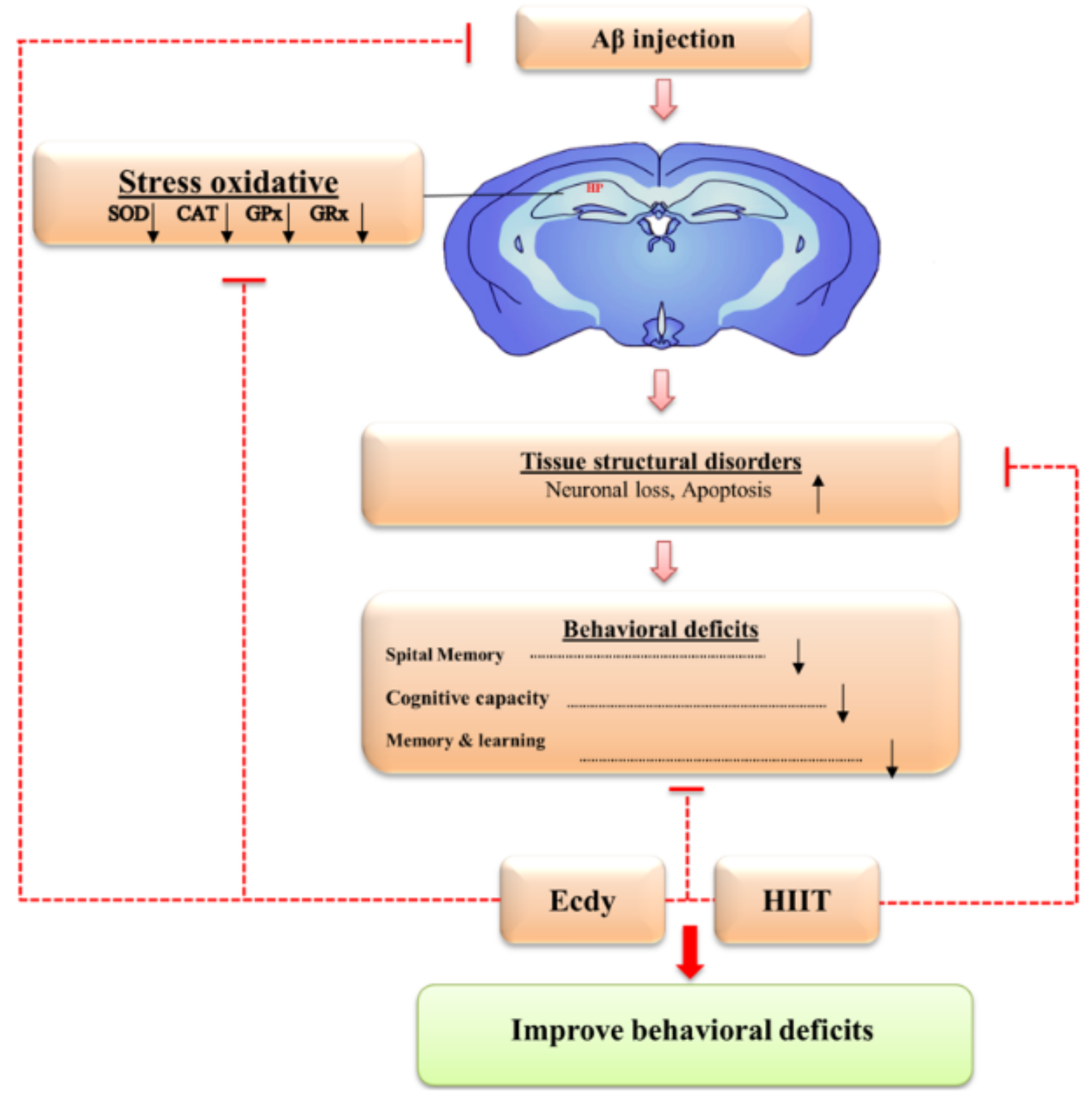

Figure 8

Schematic representation of the "protective effects of Ecdy and HIIT against AD-associated behavioral deficits in rats: Possible involved mechanisms". HP: Hippocampus; CAT: Catalase; SOD: Superoxide Dismutase; GPx: Glutathione Peroxidase; GRx: Glutathione Reductase; Ecdy: Ecdysterone; HIIT: High Intensity Interval Training. 